\title{
Descripción de Thorichthys panchovillai sp. n., una nueva especie de cíclido (Actinopterygii: Cichlidae) de la cuenca del Río Coatzacoalcos, México
}

\section{Description of Thorichthys panchovillai sp. n., a new species of cichlid (Actinopterygii: Cichlidae) from the River Coatzacoalcos Basin, Mexico}

\section{Luis Fernando Del Moral-Flores*, Eduardo López-Segovia, Tao Hernández-Arellano}

\author{
Universidad Nacional Autónoma de México (UNAM), Laboratorio de Zoología, Facultad de Estudios Superiores Iztacala. Av. de los Barrios No. 1, Los Reyes \\ Iztacala, 54090 Tlalnepantla, Estado de México, México. \\ *Autor de correspondencia \\ Email Luis Fernando Del Moral-Flores: delmoralfer@gmail.com \\ Email Eduardo López-Segovia: eduardosegovia100@gmail.com \\ Email Tao Hernández-Arellano: heraretao@hotmail.com
}

\begin{abstract}
Resumen
Thorichthys panchovillai, nueva especie, es descrita de los tributarios de la cuenca del Río Coatzacoalcos, México. La especie es diagnosticada por un conjunto de caracteres: radios de la aleta dorsal XVI, 8 - 9; radios de la aleta anal VII, $6-7$; radios de la aleta pectoral, I, $11-12$; branquiespinas totales en el primer arco branquial 11 - 12; mancha del subopérculo presente pero débilmente intensificada, en especial presenta un marcado dimorfismo sexual único y que le distingue del resto de sus congéneres: en la hembra se presenta una mancha negra entre la quinta y sexta espina dorsal.
\end{abstract}

Palabras clave: Cichliformes; ictiología; Neotrópico; sistemática; taxonomía; agua dulce; nueva especie.

\section{Abstract}

Thorichthys panchovillai, new species, is described, from distinctive of the tributaries of the River Coatzacoalcos basin, Mexico. The new species is diagnosed by a set of characters: dorsal fin rays XVI, $8-9$; anal fin rays VII, 6 - 7; pectoral fin rays, I, 11 - 12; total gill-rakers on the first branchial cleft $11-12$; subopercular stain present although weakly intensified, it exposes a notable sexual dimorphism that distinguishes it from others: the female possess a black blotch between the fifth and sixth dorsal spine.

Keywords: Cichliformes; Ichthyology; Neotropics; Systematic; Taxonomy; freshwater; new species.

Publicación registrada en Zoobank/ZooBank article registered:

LSID urn:Isid:zoobank.org:pub:6B8CE434-0C49-4AB6-9610-EC7B1EFF37C7

Acto nomenclatural/nomenclatural act:

Thorichthys panchovillai

Moral-Flores, López-Segovia \& Hernández-Arellano, 2017

LSID urn:Isid:zoobank.org:act:7009AEAB-4B4B-409C-8125-F53B2E02DF48

Citación:

Del Moral-Flores L.F., E. López-Segovia, T. Arellano-Hernández. 2017. Descripción de Thorichthys panchovillai sp. n., una nueva especie de cíclido (Actinopterygii: Cichlidae) de la cuenca del Río Coatzacoalcos México. Revista peruana de biología 24(1): 003 - 010 (Abril 2017). doi: http://dx.doi.org/10.15381/rpb.v24i1.13104
Presentado: $\quad$ 23/08/2016

Aceptado: $\quad$ 15/02/2017

Publicado online: 20/04/2017
Información sobre los autores:

LFdMF, ELS y TAH realizaron el trabajo de campo, análisis y mediciones de ejemplares. LFdMF realizó la edición del material gráfico, análisis y búsqueda de los especímenes para la comparación taxonómica, y realizó la descripción de la especie nueva. ELS y TAH intervinieron en el proceso curatorial y toma de fotografías. ELS, realizó la transparentación de ejemplares. Todos los autores participaron en la redacción del artículo. Los autores no incurren en conflictos de intereses. 


\section{Introducción}

El género Thorichthys fue descrito por Meek (1904: 222) con Thorichthys ellioti Meek 1904 (sinónimo de T. maculipinnis) como especie tipo. En general, son cíclidos de tamaño pequeño ( $<170 \mathrm{~mm}$ de SL), territoriales, de colores brillantes que se distribuyen en la vertiente Atlántica de Centroamérica: desde el centro de Veracruz, México, al sur de la cuenca del Río Montagua en Guatemala y Honduras (Miller \& Taylor 1984). Se caracterizan y distinguen de otros géneros por: ausencia de escamas en la base de la región blanda de las aletas dorsal y anal; aleta caudal lunada o truncada, en adultos los lóbulos pueden ser filamentosos; el extremo posterior de la aleta pectoral sobrepasa el origen de la aleta anal; boca terminal, región preorbital alta y con marcado perfil angular; presencia de ocho poros sensoriales desarrollados entre el mentón y el ángulo preopercular, de los cuales cinco corresponden a la región mandibular; por lo general tienen 12 vértebras precaudales y mancha subopercular en todas las especies (a excepción de T. callolepis) del género (ambos sexos) (Miller \& Nelson 1961, Miller \& Taylor 1984).

En la actualidad el género Thorichthys se encuentra representado por ocho especies válidas: T. affinis (Günther 1862) de la vertiente del Atlántico, del Río Hondo, Quintana Roo, hasta Belice y Guatemala; T. aureus (Günther 1862) de la vertiente atlántica de los ríos de Belice a Honduras; T. callolepis (Regan 1904) de la vertiente del Atlántico, cabecera y tributarios superiores del Río Coatzacoalcos; T. helleri (Steindachner 1864) de la vertiente del Atlántico, del Río Tonalá hasta Guatemala; T. maculipinnis (Steindachner 1864) de la vertiente atlántica, del Río Chachalacas al Papaloapan; T. meeki Brind 1918 de la vertiente Atlántica del Río Tonalá, pasando por Belice hasta el Petén, Guatemala; T. pasionis (Rivas 1962) vertiente atlántica de la cuenca del Río Grijalva-Usumacinta; y T. socolofi (Miller \& Taylor 1984) vertiente atlántica, en tributarios superiores de los ríos Grijalva y Usumacinta (Kullander 2003, McMahan et al. 2015). Sin embargo existe al menos una especie que permanece sin ser descrita, a la cual los acuaristas han denominado Thorichthys sp. "Mixteco" o también Thorichthys sp. "Coatzacoalcos" (Artigas-Azas 2011). Esta especie es propia de la cuenca del Río Coatzacoalcos, principalmente en los tributarios de la parte alta y media. Ya había sido observada y registrada por Regan (19061908) en San Domingo de Guzmán (actualmente la localidad se llama Santo Domingo Petapa, Oaxaca, México) y Río Sarabia, y la incluyó erróneamente dentro de Cichlosoma (=Cichlasoma) aureum (no Günther 1862). Con base en ello y para la región, trabajos posteriores la consideraron bajo esa entidad (Meek 1905, Álvarez 1950), en tanto otros, la reconocieron como una forma autóctona o afín a "Cichlasoma" aureum (Miller \& Nelson 1961, Miller 1974, Miller \& Taylor 1984, Conkel 1997). Rivas (1962) refiere a la verdadera $C$. aureum como una especie distinta a la reportada por Miller y Nelson (1961), ocasionando una adenda posterior (Miller \& Taylor 1984). Por lo anterior, el presente trabajo describe de manera formal a esta nueva entidad taxonómica.

\section{Material y métodos}

Los datos merísticos y las mediciones corporales de los organismos siguen el protocolo de Hubbs y Lagler (1958) y modificaciones referidas por Taylor y Miller (1980). Para el conteo del número de escamas de la línea lateral, se consideró la primera escama con poro por detrás del área postopercular hasta la última escama con poro de la línea superior, a la altura de esta última escama siguió el conteo en la línea inferior hasta el final del pedúnculo caudal. Las vértebras fueron contabilizadas sobre organismos diafanizados ( $\mathrm{KOH}$ al $4 \%$ ) y teńidos (Rojo de Alizarina al 0.01\%) (modificación de Wassersug 1976). Las medidas corporales se expresan como porcentaje de la longitud estándar (SL). La comparación y evaluación taxonómica de la nueva especie y sus congéneres se realizó contrastando las descripciones originales de las especies válidas, sinónimos y enmiendas taxonómicas (Meek 1904, Regan 1905, Miller \& Nelson 1961, Rivas 1962, Miller \& Taylor 1984).

Los especímenes están depositados en las siguientes instituciones: Colección Nacional de Peces, Instituto de Biología de la Universidad Nacional Autónoma de México (CNPE-UNAM); Colección Ictiológica de la Facultad de Estudios Superiores Iztacala (CIFI), UNAM; Colección de Peces, Universidad Michoacana de San Nicolás de Hidalgo, Morelia (CPUM).

\section{Resultados}

\section{Thorichthys panchovillai sp. $\mathrm{n}$.}

(Fig. 1-5, Tabla 1-2)

Cichlosoma aureum (no Günther 1862): Regan 1905, en parte: 321; Regan 1906-1908, en parte: 26. Cichlasoma aureum (no Günther 1862): Álvarez 1950, en parte: 120. Miller y Nelson 1961, en parte: 2, Tabl. 1-2, PI. 1. Thorichthys aureus (no Günther). Meek (1905: 245). Cichlasoma (Thorichthys) sp. c.f. aureum (no Günther 1862): Conkel 1997, en parte: 60. Thorichthys heller (Steindachner 1864): Kullander 2003, en parte: 644. Cichlasoma ellioti (no Meek 1904): Espinosa-Pérez et al. 1993, en parte: 66. Thorichthys ellioti (no Meek 1904): Miller 2009, en parte: 413. Thorichthys sp. aff. maculipinnis Říčan et. al. 2016.

Holotipo.- CIFI-501, macho adulto, $79.79 \mathrm{~mm} \mathrm{SL}$, colectado en el Río Almoloya, cerca del poblado de Guivisia, cuenca superior del Río Coatzacoalcos, Oaxaca, México (1657'4.33"N - 9456'58.23"W), 11 de julio de 2016, por F. Del Moral.

Paratipos.- 20 especímenes. CIFI-502, 1 hembra adulta, $47.83 \mathrm{~mm}$ SL, colectada en el Río Petapa, cerca del poblado "El Paraíso", Matías Romero, cuenca superior del Río Coatzacoalcos, Oaxaca, México (16 $\left.52^{\circ} 31.83^{\prime \prime N}-95^{\circ} 04^{\prime} 55.23^{\prime \prime W}\right), 15$ de julio de 2016, por F. Del Moral, T. Arellano y E. Segovia. CIFI 503, 1 hembra adulta, $60.1 \mathrm{~mm}$ SL, colectada en el Río El Corte, cerca de la cabecera municipal de Santa María Chimalapa, cuenca superior del Río Coatzacoalcos, Oaxaca, México (1656'36.2"N - 94³6'53.3"W), 18 de diciembre de 2014, por F. Del Moral, T. Arellano y G. Minutti. CIFI 504, 2 ejemplares: 1 macho, 45.38 $\mathrm{mm}$ SL y 1 hembra, $45.46 \mathrm{~mm} \mathrm{SL}$, colectados en el Río Almoloya, cerca del Balneario natural "El Ajal", poblado El Ajal, Barrio de la Soledad, cuenca superior del Río Coatzacoalcos, Oaxaca, México (16 $\left.46^{\circ} 01.3^{\prime \prime} \mathrm{N}-95^{\circ} 01^{\prime} 18.8^{\prime \prime W}\right), 16$ de diciembre de 2014, por F. Del Moral, T. Arellano y G. Minutti. CIFI 505, 2 ejemplares hembras, $54.17-56.42 \mathrm{~mm} \mathrm{SL}$, colectados en el Río Almoloya, cerca del Balneario natural "El Ajal", poblado El Ajal, Barrio de la Soledad, cuenca superior del Río Coatzacoalcos, Oaxaca, México (1646'01.3"N - 9501'18.8"W), 15 de julio de 2016, por F. Del Moral, E. Segovia y T. Arellano. CIFI 508, 1 ejemplar macho diafanizado, $88.31 \mathrm{~mm} \mathrm{SL}$; colectado en el Río Almoloya, Poblado "Estación Almoloya", Barrio de la Soledad, cuenca superior del Río Coatzacoalcos, Oaxaca, México (16 $\left.45^{\prime} 56.0^{\prime \prime} \mathrm{N}-95^{\circ} 03^{\prime} 07.9^{\prime \prime W}\right), 23$ de junio 

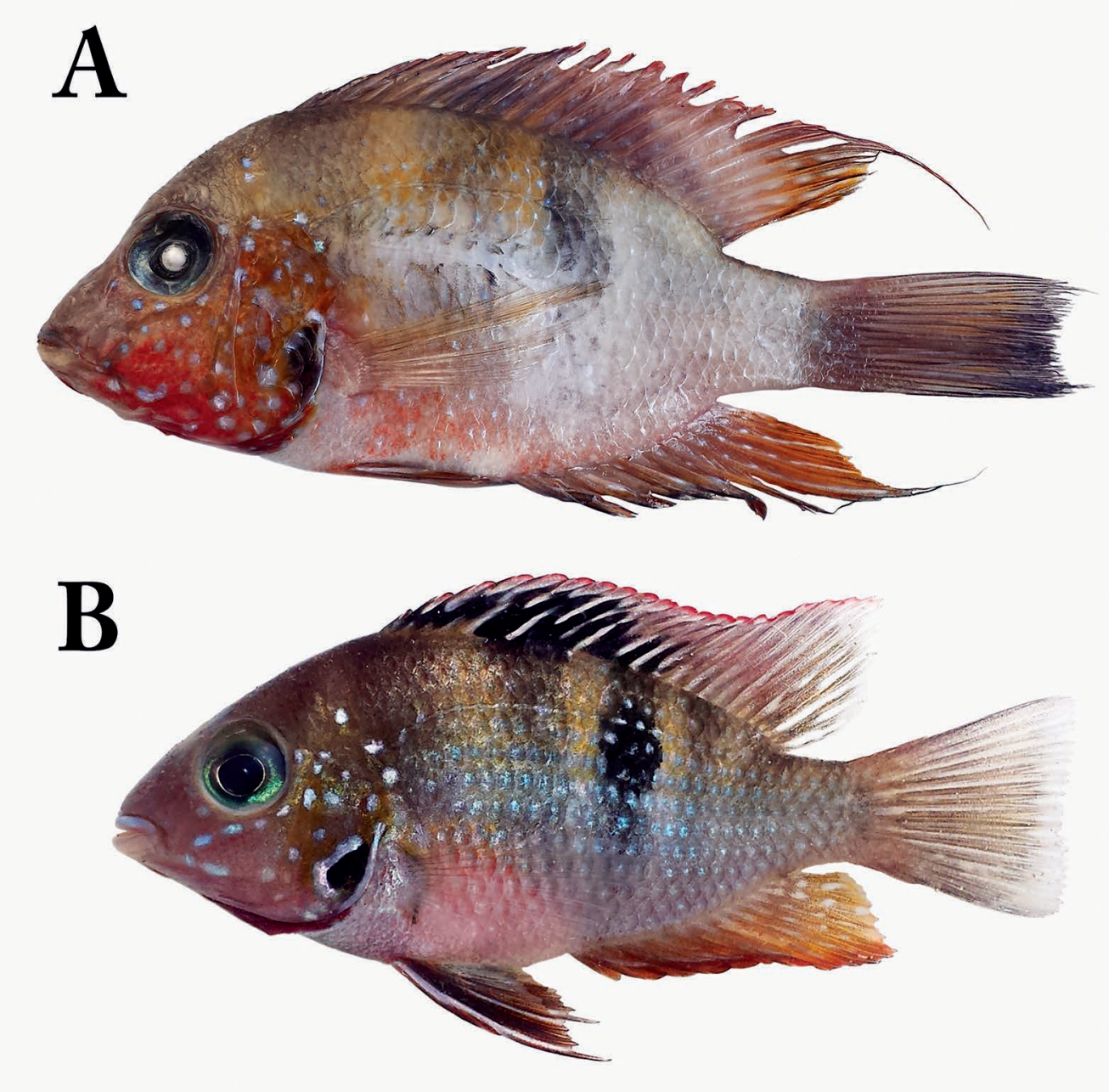

Figura 1. Thorichthys panchovillai, A) Holotipo, CIFI-501, $79.79 \mathrm{~mm} \mathrm{SL}$, macho adulto y en estado reproductivo (ejemplar fijado); B) Paratipo, CIFI-502, $47.83 \mathrm{~mm} \mathrm{SL}$, hembra adulta y en estado reproductivo (fotografía in situ).

de 2015, por F. Del Moral, T. Arellano y E. Segovia. CIFI 509, 1 ejemplar hembra diafanizado, $65.09 \mathrm{~mm} \mathrm{SL}$, colectado en el Río Almoloya, Poblado "Estación Almoloya”, Barrio de la Soledad, cuenca superior del Río Coatzacoalcos, Oaxaca, México $\left(16^{\circ} 45^{\prime} 56.0^{\prime \prime} \mathrm{N}-95^{\circ} 03^{\prime} 07.9^{\prime \prime W}\right), 23$ de junio de 2015 , por F. Del Moral, T. Arellano y E. Segovia. CNPE-IBUNAM 22273, 3 ejemplares machos, 64.69-99.0 mm SL, colectados en el Río Almoloya, cerca del poblado Guivisia, Santa María Petapa, cuenca superior del Río Coatzacoalcos, Oaxaca, México (16 $\left.57^{\circ} 19.6^{\prime \prime} \mathrm{N}-94^{\circ} 57^{\prime} 07.1^{\prime \prime W}\right), 18$ de marzo de 2016, por $\mathrm{F}$. Del Moral. CNPE-IBUNAM 22274, 2 ejemplares hembras, 46.5 - $47.62 \mathrm{~mm}$ SL, colectados en el Río Almoloya, cerca del Balneario natural "El Ajal", poblado El Ajal, Barrio de la Soledad, cuenca superior del Río Coatzacoalcos, Oaxaca, México (166'01.3"N - 9501'18.8"W), 15 de julio de 2016, por F. Del Moral, E. Segovia y T. Arellano. CPUM 9587, 4 ejemplares: 3 machos, 66.96-80.99 mm SL y 1 hembra, 59.49 mm SL, colectados en el Río Almoloya, cerca del poblado Guivisia, Santa María Petapa, cuenca superior del Río Coatzacoalcos, Oaxaca, México (1657'19.6"N - 9457'07.1"W), 18 de marzo de 2016, por F. Del Moral. CPUM 9588, 3 ejemplares: 2 machos, 41.12 - 44.54 mm SL y 1 hembra, 38.99 mm SL, colectados en el Río Almoloya, poblado "Estación Almoloya", municipio de Barrio de la Soledad, cuenca superior del Río Coatzacoalcos, Oaxaca, México (1646'17.9"N - 9501'08.6"W), 14 de diciembre de 2015, por F. Del Moral, E. Segovia, T. Arellano, U. Moreno.

Diagnosis.- Es una especie del género Thorichthys de tamaño pequeño (máx. $100 \mathrm{~mm} \mathrm{SL}$ ); y posee similitud con T. maculipinnis (Steindachner 1864). La nueva especie se distingue de sus congéneres por la siguiente combinación de características: fórmula radial dorsal, generalmente XVI, 8 o 9; fórmula radial anal VII, 6 o 7; fórmula radial pectoral, I, 11 o 12; branquiespinas totales en el primer arco branquial 11-12; mancha del subopérculo presente pero débilmente intensificada. Su coloración en vida va de tonos verde-azulados a pardo-anaranjados, se intensifica el color en la época reproductiva; coloración rojiza en las membranas branquiostegas. Dimorfismo sexual marcado y diferenciado desde etapas juveniles; la hembra presenta una mancha negra en la aleta dorsal, posicionada entre la sexta y séptima espina.

Descripción.- La forma corporal y el patrón de coloración se muestran en la figura 1 y 2 . La proporción porcentual de las mediciones corporales se muestran en la Tabla 1 ; los datos merísticos se presentan a continuación y se resumen en la Tabla 2 . Radios de la aleta dorsal: XIV(3), XVI(16); 8(5), 9(14). Radios de la 


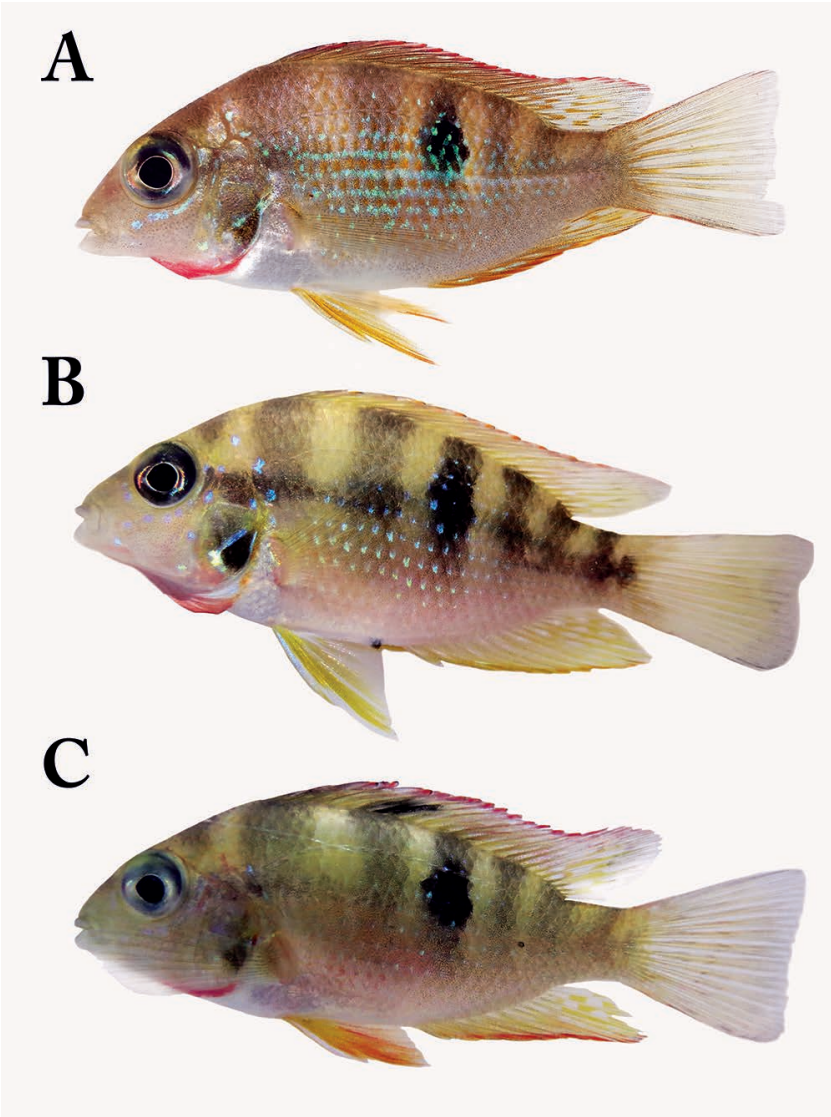

Figura 2. Thorichthys panchovillai, A) Ejemplar no tipo, CIFI510, $36.22 \mathrm{~mm} \mathrm{SL}$, macho juvenil capturado en el Poblado 14, Río Uxpanapa, Veracruz; B) Paratipo, CPUM 9588, 41.12 mm SL, macho juvenil capturado en el Río Almoloya, El Ajal; C) Paratipo, CPUM 9588, 38.99 mm SL, hembra capturada en Río Almoloya, El Ajal (fotografías in situ).

aleta anal: VII(17), VIII(2); 6(9), 7(9), 8(1). Radios de la aleta pectoral: 10(1), 11(11), 12(7). Branquiespinas del primer arco branquial: rama superior 2(9), 3(10); rama inferior 8(1), 9(18), totales 10(1), 11(8), 12 (10). Escamas en la línea lateral: 27(2), 28(14), 29(2), 30(1); escamas en la línea lateral superior: 17(1), 18(3), 19(11), 20(3), 21(1); escamas en la línea lateral inferior: 9(2), 10(7), 11(9), 13(1); escamas alrededor del pedúnculo caudal: 16(10), 17(8), 18(1). Vertebras torácicas 12, vértebras caudales 15 y totales 27 (3). Placa dental faríngea inferior de forma triangular, su ancho es ligeramente mayor que su largo (Figs. 3, 4); parte media del margen dorsal con evidente muesca a partir sus márgenes se prolongan de manera convexa hacía los extremos elongados de la placa; en los márgenes laterales hay de 28 a 30 dientes; los dientes de mayor tamaño se posicionan en la región medial y dorsal, son cónicos y algunos de forma roma.

Cuerpo oblongo y ligeramente robusto; su altura máxima se presenta por lo general en la inserción de las aletas pélvicas y se incrementa con respecto a la edad, cabe de 2 a 2.3 veces en la longitud estándar. El contorno predorsal es ligeramente convexo en juveniles y subadultos; en ejemplares machos adultos y en fase reproductiva el perfil predorsal es más pronunciado y puede ser evidente una muesca a la altura de la región preorbital. El perfil dorsal convexo, es más largo y menos pronunciado que la región predorsal, es cortado por una muesca que delimita el inicio del pedúnculo caudal cuya altura y longitud corresponden

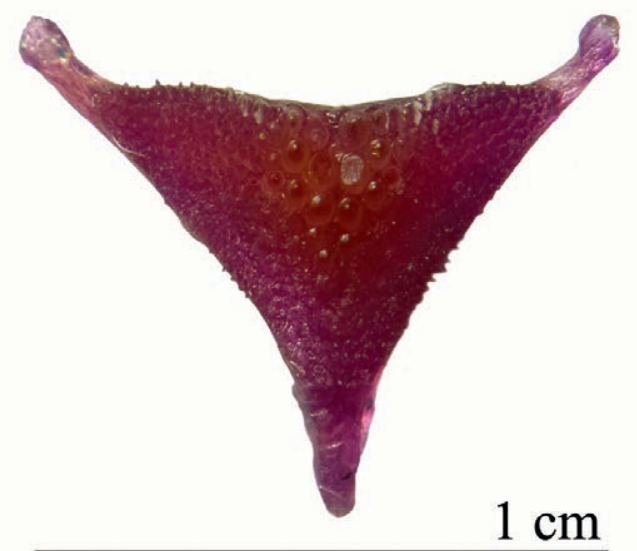

Figura 3. Thorichthys panchovillai, CIFI 514, $88.31 \mathrm{~mm} \mathrm{SL}$ placa dental faríngea inferior.

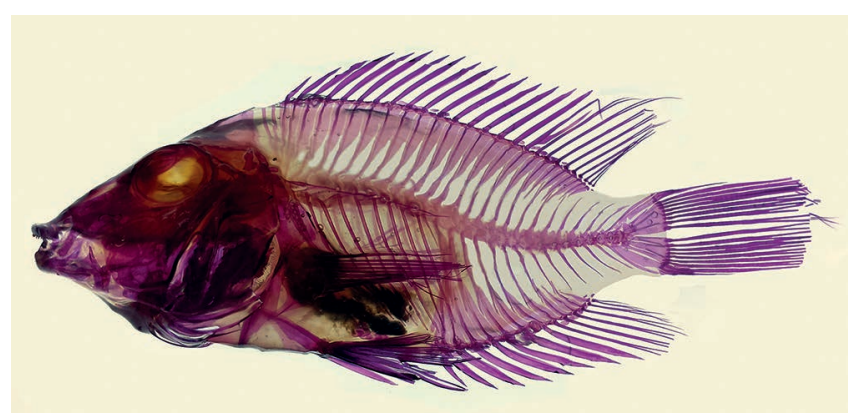

Figura 4. Thorichthys panchovillai, ejemplar tipo diafanizado, CIFI 508, macho adulto, $88.31 \mathrm{~mm}$ LS.

de 6.6 a 7.7 y 8.7 a 12 veces respectivamente en la SL. Margen ventral menos pronunciado que el dorsal, casi recto en la región prepélvica. Hocico romo y corto, cabe de 2.6 a 4 veces en la longitud cefálica. Boca pequeña y terminal, su borde superior y posterior no alcanzan respectivamente en línea recta el borde inferior y anterior de la órbita ocular. Las mandíbulas son protráctiles y tienen similar longitud. Región cefálica grande, cabe de 2.4 a 2.8 en la SL. Ojos grandes, su longitud de 2.6 a 3.5 con respecto a la longitud cefálica, su tamaño es menor en relación con la edad y talla. El ancho interorbital es menor que el diámetro ocular y es contenido 3.3 a 4.5 veces en la longitud cefálica. Branquiespinas cortas, romas y espaciadas, las basales llegan a tener forma laminar, su longitud es menor a la mitad de los filamentos branquiales.

En juveniles la aleta dorsal se origina ligeramente de una vertical trazada al final del margen opercular, mientras en los adultos se origina antes del final de la membrana opercular; su base está contenida 1.7 - 1.9 veces en la longitud estándar. La longitud de las espinas se incrementa de forma gradual, la última cabe $1.8-2.4$ veces en la longitud de la cabeza. El origen de la aleta anal sobre pasa en una línea vertical la mitad anterior de la aleta dorsal, alcanzando la base de la onceava espina; además su origen anterior sobrepasa la mancha oscura conspicua de la región dorsolateral. La parte final de los radios de las aletas dorsal y anal terminan en punta, equidistantes entre sí, en los adultos 
Tabla 1. Datos morfométricos y merísticos de la serie típica de Thorichthys panchovillai: holotipo (CIFI-501) y paratipos ( $\mathrm{n}=$ 20). Valores expresados como porcentaje de LT.

\begin{tabular}{|c|c|c|c|c|}
\hline \multirow[b]{2}{*}{ MORFOMETRÍA } & \multirow{2}{*}{$\begin{array}{c}\text { Holotipo } \\
\text { Macho } \\
\text { adulto }\end{array}$} & \multicolumn{3}{|c|}{ Paratipos } \\
\hline & & $\begin{array}{l}\text { Hembra } \\
\text { adulta } \\
\text { CIFI-502 }\end{array}$ & $\begin{array}{c}\text { Machos } \\
(\mathrm{n}=9) \\
\text { Mín - Máx (Promedio) }\end{array}$ & $\begin{array}{c}\text { Hembras } \\
(\mathrm{n}=8) \\
\text { Mín - Máx (Promedio) }\end{array}$ \\
\hline Longitud estándar, mm & 79.79 & 47.83 & $41.12-99.00(65.89)$ & $38.99-60.1(51.06)$ \\
\hline Longitud predorsal & 30.39 & 31.65 & $31.42-37.43(34.85)$ & $31.24-37.60(34.69)$ \\
\hline Longitud preanal & 61.15 & 62.78 & $57.73-65.13(62.12)$ & $60.26-67.55(64.04)$ \\
\hline Longitud prepélvica & 39.11 & 41.21 & $33.82-43.38(39.46)$ & $39.47-45.82(42.18)$ \\
\hline Altura corporal & 48.60 & 44.64 & $44.21-49.79(46.40)$ & $41.93-46.96(44.78)$ \\
\hline Longitud cefálica & 39.34 & 39.60 & $35.34-40.98$ (37.72) & $35.52-40.02(37.41)$ \\
\hline Longitud postorbital & 15.59 & 15.14 & $13.26-24.15(15.26)$ & 12.39 - $15.78(14.34)$ \\
\hline Longitud del hocico & 11.88 & 9.57 & $9.70-15.27(11.53)$ & $9.11-12.44(10.35)$ \\
\hline Ancho interorbital & 9.72 & 11.94 & $8.46-10.32(9.36)$ & $8.50-10.27(9.31)$ \\
\hline Diámetro ocular & 11.94 & 14.74 & 10.15 - $14.30(12.19)$ & 11.80 - $13.92(12.68)$ \\
\hline Longitud mandíbula superior & 6.30 & 3.33 & $5.99-9.07(7.27)$ & $5.41-7.56(6.58)$ \\
\hline Longitud del pedúnculo caudal & 11.24 & 8.34 & $8.64-11.43(10.41)$ & $8.34-12.20(10.25)$ \\
\hline Altura mínima del pedúnculo caudal & 13.79 & 14.59 & $13.53-15.02(14.03)$ & $12.91-15.53(14.26)$ \\
\hline Longitud de la base de la aleta dorsal & 57.54 & 56.49 & $51.96-60.26(55.57)$ & $51.65-56.81(54.34)$ \\
\hline Longitud de la última espina dorsal & 19.45 & 17.58 & $17.47-20.74(19.08)$ & $14.76-20.78(18.24)$ \\
\hline Longitud base de la aleta anal & 29.53 & 30.59 & $24.11-28.34(26.36)$ & $23.27-29.17(26.69)$ \\
\hline Longitud de la aleta pectoral & 35.93 & 33.39 & $28.80-36.28(34.09)$ & $30.66-36.49(33.73)$ \\
\hline Longitud de la aleta pélvica & 26.47 & 26.09 & $20.52-33.97(26.55)$ & $22.79-29.33(26.89)$ \\
\hline
\end{tabular}

pueden tener terminaciones filamentosas. Las aletas pectorales son grandes, 2.7 - 3.4 veces en la longitud estándar, de igual o similar longitud que la región cefálica $(0.9-1.1$ veces en la misma); tiene forma asimétrica, ligeramente emarginada, con terminación puntiaguda la cual en adultos y en estado reproductivo puede ser filamentosa. Origen de las aletas pélvicas por delante de la vertical trazada desde la axila pectoral, su longitud menor que las pectorales y está contenida 2.9 a 4.3 veces en la longitud estándar; de forma triangular y terminación puntiaguda en el primer radio el cual puede presentar una prolongación filamentosa en la fase reproductiva. Aleta caudal emarginada, en algunos machos adultos en fase de reproducción, pueden presentar ligeras extensiones filamentosas en los radios marginales.

Coloración en alcohol.- Los ejemplares preservados son de color pardo en la región dorso-lateral, disminuyendo su intensidad hacia la región abdominal, donde las tonalidades varían de amarillas, grises o blanquizcas. En algunos ejemplares adultos se

Tabla 2. Variación intraespecífica del número de espinas y radios de la aleta dorsal y anal y de branquiespinas de algunas especies del género Thorichthys. Los datos de T. scolofi fueron tomados de Miller y Taylor (1984).

\begin{tabular}{|c|c|c|c|c|c|c|c|c|c|c|c|c|}
\hline DORSAL & XIV & $\mathrm{XV}$ & XVI & XVII & XVIII & & 7 & 8 & 9 & 10 & 11 & 12 \\
\hline T. panchovillai & & 3 & 16 & & & & & 5 & 14 & & & \\
\hline T. callolepis & & 7 & 29 & & & & & 13 & 20 & 3 & & \\
\hline T. maculipinnis & & 3 & 12 & 1 & & & 2 & 4 & 10 & & & \\
\hline T. meeki & & & 6 & & & & & 3 & 3 & & & \\
\hline T. helleri & & 4 & & & & & & & & 2 & 2 & \\
\hline T. socolofi & & 4 & 51 & 5 & & & & 19 & 41 & & & \\
\hline T. pasionis & 1 & 36 & & & & & & 30 & 7 & & & \\
\hline ANAL & VI & VII & VIII & IX & & & 6 & 7 & 8 & 9 & 10 & \\
\hline T. panchovillai & & 17 & 2 & & & & 9 & 9 & 1 & & & \\
\hline T. callolepis & 5 & 30 & 1 & & & & 20 & 16 & & & & \\
\hline T. maculipinnis & & 8 & 8 & & & & 4 & 11 & & 1 & & \\
\hline T. meeki & & & 1 & 5 & & & & 6 & & & & \\
\hline T. helleri & & 4 & & & & & & 3 & 1 & & & \\
\hline T. socolofi & & 4 & 55 & 1 & & & 51 & 9 & & & & \\
\hline T. pasionis & & 1 & 36 & & & & & 31 & 6 & & & \\
\hline BRANQUIESPINAS & & 10 & 11 & 12 & 13 & 14 & 15 & 16 & 17 & 18 & 19 & \\
\hline T. panchovillai & & 1 & 8 & 10 & & & & & & & & \\
\hline T. callolepis & & & 2 & 19 & 10 & 4 & & & & & & \\
\hline T. maculipinnis & & & 5 & 11 & & & & & & & & \\
\hline T. meeki & & & & & & & & 3 & 2 & 1 & & \\
\hline T. helleri & & & & 4 & & & & & & & & \\
\hline T. socolofi & & 1 & 8 & 42 & 8 & 1 & & & & & & \\
\hline T. pasionis & & & & & & & & 1 & 23 & 10 & 3 & \\
\hline
\end{tabular}




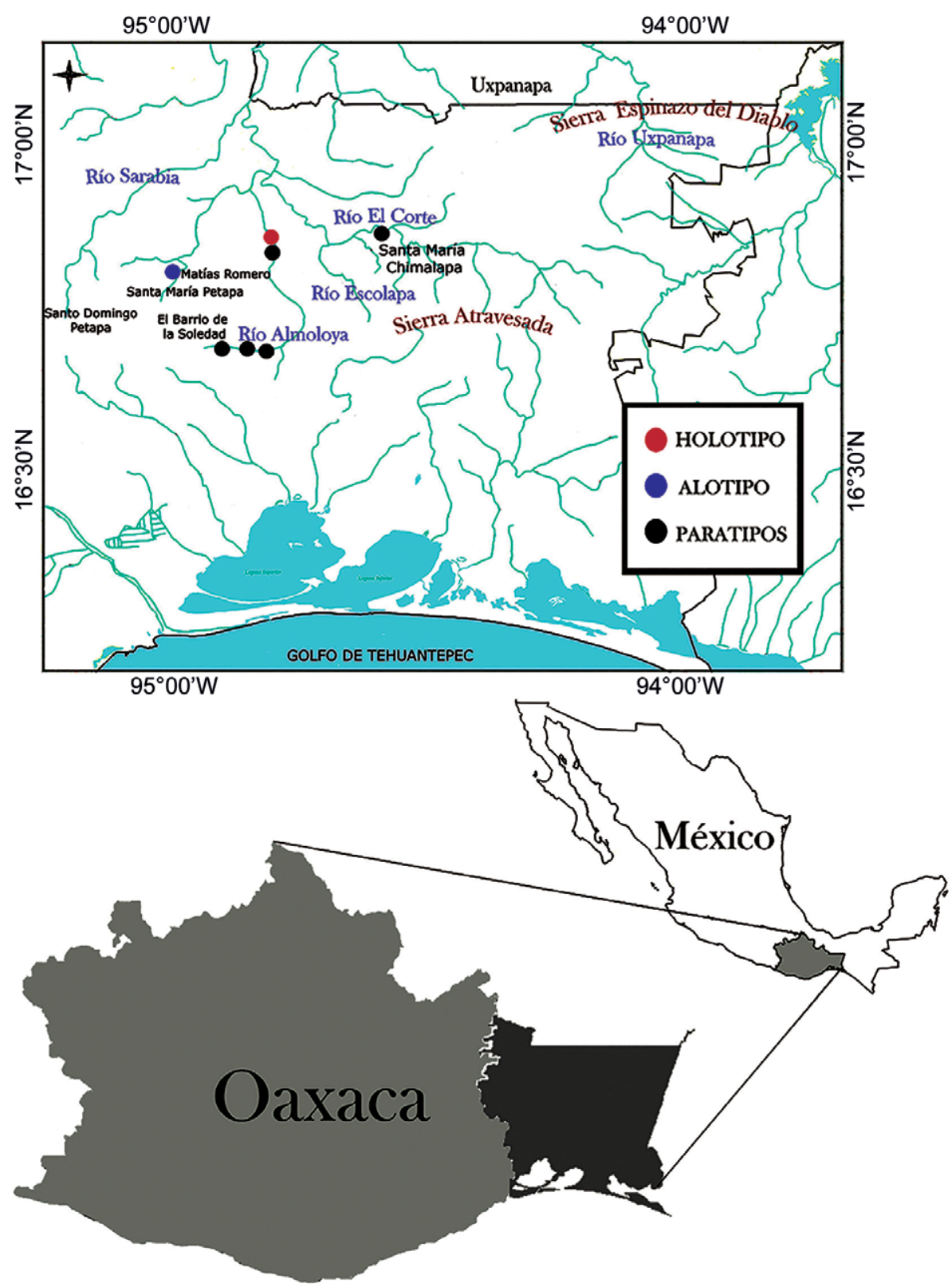

Figura 5. Mapa del área de distribución de Thorichthys panchovillai. Círculo rojo representa la localidad tipo.

puede apreciar, aún en el estado de conservación, la coloración rojiza y anaranjada típica del estado reproductivo (Fig. 1A). Tanto en vida como en estado de conservación se observan 7 barras verticales oscuras espaciadas en los costados del cuerpo, la última simula una mancha en el pedúnculo caudal, su intensidad es variable y son más notorias en la región dorsal en contraste con la región ventral donde suelen desaparecer a la altura de la parte media de las aletas pectorales. La primera barra se origina por delante del origen de la aleta dorsal, se extiende por detrás del área superior preopercular, su contorno anterior es convexo mientras el posterior ligeramente cóncavo y se origina cerca de la segunda y tercer espina dorsal. En la tercera barra se presenta una mancha oscura, bordeada por puntos de color azul-iridiscente y de ancho similar al área entre la línea lateral superior e inferior, dando una aparente mayor intensidad de esta barra. En algunos ejemplares se presenta una línea oscura, de variable intensidad, cuyo origen corresponde al margen posterior del área orbital y se extiende hasta la última barra del pedúnculo caudal (Fig. 2B).
Coloración en vida.- La coloración del fondo de los organismos en vida presenta variación dependiendo del área geográfica donde habiten, grado de madurez e incluso la hora del día en que se capturen. Sin embargo las tonalidades van de azules, verdes y anaranjados. En las regiones de la mejilla, nuca, área opercular y flancos corporales se encuentran salpicados por pequeńas manchas azul iridiscentes, dispuestas en un patrón complejo, el cual se vuelve más intenso durante la fase reproductiva y en organismos machos. Existe una mancha subopercular oblicua de intensidad variable, más conspicua en estado adulto, esta bordeada por una franja azul iridiscente. En la región inferior de la región cefálica, principalmente en las membranas branquiostegas, se presenta una coloración rojizo-anaranjada cuya intensidad y área depende del grado de madurez y sexo.

Las aletas pectorales y caudal de color hialino, sin embargo en machos tienden a tener un tinte amarillo siendo más intenso con la edad, alcanzado cierto grado de color negro. Aleta dorsal 
de color pardo, siendo más intensa la región anterior; su margen dorsal de color rojizo-anaranjado; en su parte media las hembras presentan una mancha negra entre la quinta y sexta espina dorsal, en época reproductiva la mancha es más evidente y cubre gran parte del área espinosa de la aleta dorsal (Figs. 1B, 2C). Aleta anal de color anaranjado y amarillo, con un patrón de manchas oblicuas traslucidas, su borde marginal rojizo. Aletas pélvicas de mayor intensidad en coloración que el resto, de amarillo a oscuro, y principalmente entre la membrana que separa el primer y tercer radio; primer espina y prolongación filamentosa, cuando presente, están bordeadas por una línea de color azul iridiscente.

Etimología: El epíteto específico es un nombre hipocorístico del histórico, jefe y pilar fundamental de la revolución mexicana, José Doroteo Arango Arámbula, mejor conocido como Francisco Villa cuyo alías corresponde a "Pancho Villa".

Distribución: Thorichthys panchovillai, se encuentra distribuida ampliamente en los tributarios del Río Coatzacoalcos, incluyendo los de la cuenca superior (Río Almoloya (=Ajal), La Aurora, entre otros), región oriental (Uxpanapa) y parte media y baja propios de la planicie. Es una especie endémica del Río Coatzacoalcos, México (Fig. 5). Se encuentra asociada en simpatria con otras especies de cíclidos, entre ellas: su congénere Thorichthys callolepis, Maskaheros regani, Paraneetroplus bulleri, Trichromis salvini y Vieja sp. (Miller \& Nelson 1961, Miller 1974).

\section{Discusión}

Thorichthys panchovillai, puede distinguirse de todas las especies del género, por la presencia de una mancha negra en la aleta dorsal en las hembras, carácter único de la especie, representando un marcando y evidente dimorfismo sexual.

De acuerdo con Miller y Taylor (1984), dentro del género Thorichthys se pueden reconocer dos subgrupos con base en sus características anatómicas y merísticas. El subgrupo denominado T. helleri (integrado por T. helleri, T. callolepis y T. maculipinnis), se caracteriza por tener de 10 a 14 branquiespinas, de VII A VIII espinas en la aleta anal, mandíbula inferior no proyectada por lo general más allá de la superior, de 6 a 12 dientes centrales en la placa faríngea inferior, entre otros.

La nueva especie está relacionada con los integrantes del subgrupo de T. helleri (sensu Miller \& Taylor 1984). El congénere más similar con $T$. panchovillai es $T$. maculipinnis, con el cual comparte una moda similar en relación a los valores merísticos dorsales, anales y branquiales, a pesar de ambas especies presentaren una distribución alopátrica, la primera es restringida a la cuenca del Río Coatzacoalcos y la segunda a la cuenca del Río Papaloapan. Además, es posible distinguir a T. panchovillai de T. maculipinnis por presentar un dimorfismo sexual único (hembra con mancha en la aleta dorsal), ojos más grandes (2.6 - 3.5 veces en la longitud cefálica), ancho interorbital menor al diámetro ocular (en T. maculipinnis el ancho interorbital es igual al diámetro ocular).

Thorichthys panchovillai difiere de T. helleri por tener un menor número de radios dorsales, de 8 a 9 (en comparación a 10 - 11); además esta última habita en la cuenca del Río Grijalva-Usumacinta). La única especie del género que vive en simpatría con la nueva especie es $T$. callolepis, de la cual se diferencian fácilmente porque está última no tiene la mancha subopercular típica de T. panchovillai.
El resto de sus congéneres se diferencian de la nueva especie por presentar los siguientes caracteres: una mancha basicaudal evidente, menor número de espinas dorsales (XIV - XV) y número elevado de branquiespinas $(16-19)$ totales del primer arco branquial en T. pasionis; mayor número de radios dorsales $(10$ - 11), espinas anales (VIII - IX) y branquiespinas (16 18) en T. meeki; finalmente, mayor número de espinas dorsales (XV - XVII), en promedio un mayor número de espinas anales (VII - IX, moda en VIII) y una mayor variación en el número de branquiespinas $(10-14)$ en $T$. socolofi.

En la actualidad los estudios filogenéticos, en ciertos grupos (e.g., Heroini) de la familia Cichlidae con distribución en el continente americano, basados tanto en datos morfológicos y moleculares revelan diversos aspectos acerca de los patrones de diversificación. Es así como se han descrito nuevos géneros, esclarecido ciertos problemas taxonómicos, establecido sus relaciones de parentesco y de distribución en Centro y Sudamérica (e.g., McMahan et al. 2015; Říčan et al. 2016).

Como se aprecia, es necesario seguir realizando estudios que contribuyan al conocimiento de la ictiodiversidad mexicana.

Clave taxonómica para las especies del género Thorichthys:

1A. Branquiespinas 10-14; espinas anales VII-VIII, rara vez IX 2

1B. Branquiespinas 14-22; espinas anales, VIII-X, rara vez VII 7

2A. Sin mancha subopercular

T. callolepis

2B. Con mancha subopercular

3

3A. Aleta dorsal predominantemente con XV espinas y 10 o más radio

T. belleri

3B.Aleta dorsal con XVI espinas y por lo general 9 o menos radio dorsales 4

4A. Con patrón oscuro prominente en los costados aparentando una "L" invertida

T. socolofi

4B. Sin el patrón oscuro como el descrito anteriormente

5

5A. Menos de 10 branquiespinas en el primer arco brnaquial; por lo general XVI espinas dorsales; longitud de la última espina dorsal $\leq 1.5$ en la longitud cefálica

T. aureus

5B. Más de 11 branquiespinas en el primer arco branquial

6

6A. Ancho interorbital menor al diámetro ocular; hembra con mancha oscura entre la 6 y 7 espina dorsal T. panchovillai

6B. Ancho interorbital igual al diámetro ocular; hembra sin mancha entre la 6 y 7 espina dorsal

T. maculipinnis

7A. Con mancha oval basicaudal

T. pasionis

7B. Sin mancha oval en el pedúnculo caudal 8

8A. Aleta pectoral no extendida hasta el nivel de la última espina anal, premaxilar extendido hasta $1 / 4$ de la longitud del ojo T. meeki

8B. Aleta pectoral extendida hasta el nivel de la última espina anal, premaxilar no extendido más allá del margen anterior del ojo

T. affinis

Material comparativo.- Thorichthys panchovillai. CIFI 510, 1 ejemplar macho juvenil, $36.22 \mathrm{~mm} \mathrm{SL}$, colectado en el Poblado 14, Río Uxpanapa, Uxpanapa, cuenca del Río Coatzacoalcos, Veracruz, México, el 12 de julio de 2016, por F. Del Moral, T. Arellano y E. Segovia. CIFI 514, 1 ejemplar macho adulto, $88.31 \mathrm{~mm}$ SL, colectado en Monte Oscuro, Matías Romero Avendańo, Oaxaca, México (1559'01.7"N 97²1'12.7"W), 7 de agosto de 2015, por F. Del Moral. Thorichthys callolepis $(\mathrm{n}=36)$. CIFI-25, 14 ejemplares, colectados en el Río Almo- 
loya, cerca del poblado Guivisia, Santa María Petapa, cuenca superior del Río Coatzacoalcos, Oaxaca, México (1657'19.6"N - 9457'07.1"W), 17 de diciembre de 2015, por F. Del Moral. CIFI-79, 5 ejemplares, colectados en el Río El Corte, cerca de la cabecera municipal de Santa María Chimalapa, cuenca superior del Río Coatzacoalcos, Oaxaca, México (1656'36.2"N - 94³6'53.3"W), 18 de diciembre de 2014, por F. Del Moral, T. Arellano y G. Minutti. CIFI-126, 4 ejemplares, colectados en el Río Los Milagros, cerca de la cabecera municipal de Santa María Chimalapa, cuenca superior del Río Coatzacoalcos, Oaxaca, México (1653'53.53", W 94³9'25.75"W), el 8 de agosto de 2015, por F. Del Moral, T. Arellano y E. Segovia. CIFI-297, 11 ejemplares, colectados en el Río Almoloya, cerca del poblado Guivisia, Santa María Petapa, cuenca superior del Río Coatzacoalcos, Oaxaca, México (16²57'19.6"N - 94º57'07.1"W), 18 de marzo de 2016, por F. Del Moral. CIFI-499, 2 ejemplares, colectados en el Río El Pinal, cerca de la cabecera municipal de Santa María Chimalapa, cuenca superior del Río Coatzacoalcos, Oaxaca, México (1656'36.2"N - 94²36'53.3"W), 24 de junio de 2015, por F. Del Moral, T. Arellano y E. Segovia. Thorichthys belleri $(n=4)$. CNPE-IBUNAM 16702, 2 ejemplares, colectados en Laguna Punteda en Salsipuedes, Reserva de la Biosfera Pantanos de Centla, Río Usumacinta, Tabasco, México, 8 de diciembre de 2002. CNPE-IBUNAM 16718, 2 ejemplares, colectados en Laguna San Isidro en Salsipuedes, Reserva de la Biosfera Pantanos de Centla, Río Usumacinta, Tabasco, México, el 24 de junio de 2001. Thorichthys maculipinnis $(\mathrm{n}=16)$. CNPE-IBUNAM 20023, 11 ejemplares, colectados en Río Apazapan, Ignacio de la Llave, Veracruz, México, el 5 de julio de 2007. CNPE-IBUNAM 2450, 3 ejemplares, colectados en el Río Mancuernas cerca del Río la Antigua, Puente Nacional, Veracruz, México, 13 de enero de 1985. CNPE-IBUNAM 16890, 2 ejemplares, colectados en el Río Zapote, Cuitlahuac, Veracruz, México, 19 de septiembre de 2011. Thorichthys pasionis $(\mathrm{n}=37)$. CNPE-IBUNAM 1258, 1 ejemplar, colectado en la Laguna "El Chiribital”, Villahermosa, Tabasco, México, 11 de abril de 1982. CNPE-IBUNAM 1268, 1 ejemplar, colectado en la Laguna "El Chiribital", Villahermosa, Tabasco, México, el $11 \mathrm{de}$ abril de 1982. CNPE-IBUNAM 6575, 32 ejemplares, colectados en Aguada, Ix-Bakab, Campeche, México, el 17 de abril de 1988. CNPE-IBUNAM 12181, 3 ejemplares, colectados en la Laguna "Horizonte", Nacajuca, México, 24 de enero de 1998. Thorichthys meeki ( $\mathrm{n}=6$ ). CNPE-IBUNAM 6248, 6 ejemplares, colectados en laguna cerca de la zona industrial de Chetumal, Quintana Roo, México, 29 de mayo de 1987.

\section{Agradecimientos}

Agradecemos el apoyo en campo a los biólogos G. Minutti, U. Moreno, C. Calderón, S. Ballesteros, K. Beltrán, F. Aranda, E. León, M. Guadarrama, C. Servín, J. De La Cruz, E. Rubio y K. Solís; a la señora Felicitas y a F. Jiménez Zarate y familia cuyo apoyo durante el muestreo fue fundamental para el buen término de la presente contribución. A H. Espinosa Pérez por el acceso al material comparativo alojado en la CNPE-IBUNAM. Finalmente a la Biol. Andrea Colis por su ayuda técnica y corrección de estilo.

\section{Literatura citada}

Álvarez J. 1950. Clave para la determinación de especies en los peces de las aguas continentales mexicanas. Ciudad de México, Secretaría de Marina, Dirección General de Pesca e Industrias Conexas.

Artigas-Azas J.M. 2011. Thorichthys - Mayaskatter som lever under vattnet. Ciklidbladet 44(1): 6-13.

Conkel D. 1997. Cichlids of North \& Central America. New Jersey: T.F.H. Publications, Inc., Neptune City.

Espinosa-Pérez, H., M.T. Gaspar-Dillanes \& P. Fuentes-Mata. 1993. Listados faunísticos de México III. Los peces dulceacuícolas mexicanos. Ciudad de México: Instituto de Biología, Universidad Nacional Autónoma de México.

Hubbs C.L. \& K.F. Lagler. 1958. Fishes of the Great Lakes region. 2nd edn. Michigan: Cranbrook Institute of Science, Bloomfield Hills.

Kullander S.O. 2003. Family Cichlidae (Cichlids): In: R.E. Reis, S.O. Kullander and C.J. Ferraris Jr., eds. Checklist of the Freshwater Fishes of Central and South America. Porto Alegre: EDIPUCRS. Pp. 605-654.

Meek S.E. 1904. The fresh-water fishes of Mexico north of the isthmus of Tehuantepec. Field Columbian Mus., Zool. ser. 5: 1-252. http://dx.doi.org/10.5962/bhl.title.2229.

Meek S.E. 1905. A collection of fishes from the Isthmus of Tehuantepec. Proc. Biol. Soc. Wash. 18, 243-245.

McMahan C.D., W.A. Matamoros, K.R. Piller \& P. Chakrabarty. 2015. Taxonomy and systematics of the herichthyins (Cichlidae: Tribe Heroini), with the description of eight new Middle American genera. Zootaxa 3999 (2): 211-234. http://dx.doi. org/10.11646/zootaxa.3999.2.3.

Miller R.R. 1974. Cichlasoma regani, a new species of Cichlid fish from the Rio Coatzacoalcos basin, Mexico. Proc. Biol. Soc. Wash. 87(40): 465-472.

Miller R.R. 2009. Peces dulceacuícolas de México. Ciudad de México: Comisión Nacional para el Conocimiento y Uso de la Biodiversidad, Sociedad Ictiológica Mexicana A. C., El colegio de la Frontera Sur y Consejo de los Peces del Desierto México-Estados Unidos.

Miller R.R. \& B.C. Nelson. 1961. Variation, life colors, and ecology of Cichlasoma callolepis, a cichlid fish from southern Mexico, with a discussion of the Thorichthys species group. Occas. Pap. Mus. Zool. Univ. Mich., 622: 1-9.

Miller R.R. \& J.N. Taylor. 1984. Cichlasoma socolofi, a new species of cichlid fish of the Thorichthys group from northern Chiapas, Mexico. Copeia 4: 933-940.

Regan C.T. 1905. A revision of the fishes of the American cichlid genus Cichlosoma [sic] and of the allied genera. Ann. Mag. Nat. Hist., ser. 7, 16: 316-340.

Regan C.T. 1906-08. Pisces, in: Biologia Centrali-Americana. R.H. Porter, Londond, pp. 8: 1-203.

Říčan O., L. Piálek, K. Dragová \& J. Novák. 2016. Diversity and evolution of the Middle American cichlid fishes (Teleostei: Cichlidae) with revised classification. Vertebr. Zool. 66(1): 1-102.

Rivas L.R. 1962. Cichlasoma pasionis, a new species of cichlid fish of the Thorichthys group, from the Rio de la Pasion, Guatemala. Q. J. Fla. Acad. Sci. 25(2):147-156.

Taylor J.N. \& R.R. Miller. 1980. Two new fishes, genus Cichlasoma, from Chiapas, Mexico. Occ. Pap. Mus. Zool. Univ. Mich. 693: $1-16$

Taylor, W. R. y Van Dyke G. C. (1985). Revised procedures for staining and clearing smallfishes and other vertebrates for bone and cartilage study. Cybium, 9 (2): 107-119.

Wassersug R.J. 1976. A procedure for differential staining of cartilage and bone in whole formalin-fixed vertebrates. Stain Technol. 51(2): 131-134. 